\title{
Supervivencia de Molares Temporales con Tratamiento de Pulpotomía. Un Análisis de Kaplan-Meier
}

\author{
Survival of Deciduous Molar Teeth with Pulpotomy Treatment. A Kaplan-Meier Analysis
}

\author{
Cristóbal Salinas*; Luigi Guidotti'; Carolina Rodríguez"* \& Cristina Sotomayor ${ }^{* * *}$
}

SALINAS, C.; GUIDOTTI, L.; RODRÍGUEZ, C. \& SOTOMAYOR, C. Supervivencia de molares temporales con tratamiento de pulpotomía. Un análisis de Kaplan-Meier. Int. J. Odontostomat., 7(3):441-446, 2013.

RESUMEN: El objetivo de este estudio es estimar la supervivencia de molares temporales sometidos a pulpotomía en la clínica odontológica de la Universidad Austral de Chile (UACh) y su relación con las variables: edad, sexo, y ubicación según arco. Se realizó una cohorte retrospectiva, de carácter censal, en la cual se revisaron las fichas clínicas de pacientes de 4 a 10 años atendidos en la clínica UACh, sometidos a tratamiento de pulpotomía desde marzo de 2008 hasta Junio 2011. Se incluyeron 125 pacientes, con un total de 170 pulpotomías. Se realizó un examen clínico en aquellos pacientes en los cuales la pulpotomía aún no había fracasado, aquellos pacientes que no asistieron al examen clínico, fueron censurados a la fecha del último control consignado en la ficha clínica. La tabulación y análisis de datos se realizó mediante el programa estadístico IBM SPSS Statistics 19. La supervivencia se obtuvo con el método Kaplan-Meier. Para determinar la diferencia estadística entre los intervalos de edad, sexo y ubicación según arco se utilizó el test de Mantel-Cox logrank $(p<0,05)$. La mediana de supervivencia general es de 32 meses, no existe diferencia estadísticamente significativa entre los intervalos de edades estudiados, el sexo y los arcos dentarios. El pronóstico de la pulpotomía es bueno ya que establece que existe $50 \%$ de probabilidad de que el diente permanezca en boca al menos 32 meses, cumpliendo con el objetivo de evitar lapérdida prematura y sus consecuencias.

PALABRAS CLAVE: pulpotomía, dientes temporales, análisis de supervivencia.

\section{INTRODUCCIÓN}

La Organización Mundial de la Salud (OMS) ha estimado que entre el 60 y $90 \%$ de los niños del mundo presentan lesiones de caries con cavitación evidente. Esta enfermedad tiene la capacidad de exponer la pulpa (Koshy \& Love, 2004) y provocar un estado infeccioso que podría afectar al germen del diente permanente y estructuras adyacentes (Waterhouse et al., 2000).

La caries dental es la razón más prevalente de pérdida prematura de dientes temporales (Koshy \& Love), lo que podría provocar la pérdida de la integridad del arco dentario y posibles maloclusiones (Fuks, 2008) y alteraciones fonéticas (Cordeiro \& Rocha, 2005).

El más común de los tratamientos pulpares en dentición temporal es la pulpotomía (Huth et al., 2005,
Fuks, 2008). El procedimiento consiste en remover la pulpa coronal con sintomatología reversible, en caso de lesiones cariosas profundas cercanas a la pulpa (Alaçam et al., 2009), exposiciones pulpares accidentales o producto de traumatismos (Fuks, 2000), con el fin de mantener la vitalidad de la pulpa, en su porción radicular, de manera de lograr mantener el diente en boca hasta su exfoliación natural (Cordeiro \& Rocha).

En cuanto a las tasas de éxito de las pulpotomías respecto a los diferentes medicamentos utilizados (formocresol, sulfato férrico, agregado de trióxido mineral MTA) y a la técnica con láser, estas han probado ser similares exceptuando el hidróxido de calcio que presenta resultados inferiores (Fuks, 2008; Huth et al.). Hill (2007) hace una estimación de la duración de la terapia de pulpotomía analizando factores que la pudiesen afectar; obteniendo una media-

\footnotetext{
Instituto de Odontoestomatología, Facultad de Medicina, Universidad Austral de Chile, Valdivia, Chile.

** Docente Instituto de Odontoestomatologia, Facultad de Medicina, Universidad Austral de Chile, Valdivia, Chile.

${ }^{* * *}$ Docente Instituto Salud Pública, Facultad de Medicina, Universidad Austral de Chile, Valdivia, Chile.
} 
na de supervivencia de $32,7 \pm 1,4$ meses para dientes vitales.

Actualmente no existe evidencia nacional respecto de la duración del tratamiento de un diente bajo un protocolo estándar, por lo tanto el objetivo de este trabajo es analizar la supervivencia de las terapias pulpares vitales realizadas en la clínica Odontológica UACh bajo un protocolo estandarizado con el método de Kaplan-Meier y su relación con las variables: sexo, edad y ubicación según arco dentario con el método Mantel-Cox logrank.

\section{MATERIAL Y MÉTODO}

El diseño del estudio corresponde a una cohorte retrospectiva para analizar el tiempo de supervivencia de las pulpotomías realizadas en niños entre 4 y 10 años atendidos en la Clínica Odontológica UACh entre el 01 de marzo de 2008 y 30 de Junio de 2011. Los criterios de elegibilidad fueron pacientes pediátricos a los que se les realizó pulpotomía en molares temporales vitales con caries profundas cercanas a la pulpa con o sin perforación pulpar accidental; con sintomatología de pulpitis reversible; tratadas con sulfato férrico; con consentimiento informado de los padres; excluyendo aquellos dientes de pacientes donde se realizó pulpotomía debido a exposiciones pulpares por trauma.

El estudio fue de carácter censal, ya que consideró la totalidad de pacientes que cumplen con los criterios formulados. La unidad de estudio corresponde a pacientes con dientes tratados con pulpotomía. La variable dependiente es el tiempo en meses de supervivencia en boca de los dientes tratados con pulpotomía y su relación con las variables sexo, edad y ubicación según arco.

La recolección de datos se realizó mediante la revisión de fichas odontopediátricas de pacientes atendidos en la Clínica Odontológica UACh por dos revisores entre los meses de Marzo y Mayo de 2012; en caso de existir registro de fracaso clínico, la variable tiempo de supervivencia se obtuvo considerando los meses que duró el tratamiento, de lo contrario, se realizó la corroboración de su permanencia en boca mediante examen clínico de rutina durante Mayo de 2012 por un investigador elegido previamente al azar (lanzando una moneda). Aquellos pacientes que presentaron alguna alteración o patología bucodentaria fue- ron derivados oportunamente a atención dentro de la misma clínica.

El análisis descriptivo de los datos fue realizado con el software IBM SPSS Statistics 19 (IBM., Chicago, EE.UU); el análisis de supervivencia se basó en el método Kaplan-Meier, considerando como dato censurado todos los casos en que el molar se encontrase presente; con ausencia de: dolor, movilidad patológica, sensibilidad a la palpación, e inflamación vestibular o fístula; y aquellos casos en que el diente exfolió naturalmente. Los pacientes que no asistieron al control para la realización del estudio se censuraron con la fecha del último control realizado en la Universidad. Aquellos casos en que el diente exfolió naturalmente entre el último control y el examen clínico del estudio se censuró a la fecha de este último.

Se construyeron tablas de estadísticas resumen, además de curvas de supervivencia y el test MantelCox logrank, para describir y comparar las variables analizadas. La significancia estadística fue considerada con un valor de $P<0,05$. El reporte de esta investigación se ha realizado según las recomendaciones de la declaración STROBE. (Von Elm et al., 2007). Los recursos materiales fueron aportados por la Universidad Austral de Chile y no se recibió ningún aporte financiero de parte de entidades externas a la Universidad.

\section{RESULTADOS}

Se revisó un total de 1.357 fichas clínicas; de estas, 126 cumplían con los criterios de elegibilidad (9,3\%); 1 paciente no aceptó participar el estudio, el resto aportó con 170 casos de pulpotomía. En las fichas clínicas 21 casos presentaban fracaso, se controlaron clínicamente 73 pulpotomías y 76 tratamientos se censuraron a la fecha del último control consignado en la ficha clínica (Fig. 1).

Del total de 125 pacientes evaluados, el $51,2 \%$ fueron mujeres y el $48,8 \%$ hombres; el número de tratamientos realizados alcanzó un 45,3\% (77/170) y un $54,7 \%(93 / 170)$ respectivamente. La mediana de los meses de seguimiento fue de 9 meses (rango 0 - 43 meses).

La edad media fue de 7,07 $\pm 1,76$ años, con una moda de 8 años; según sexo la edad media fue de 7,21 $\pm 1,92$ años en varones y 6,94 $\pm 1,61$ años en 


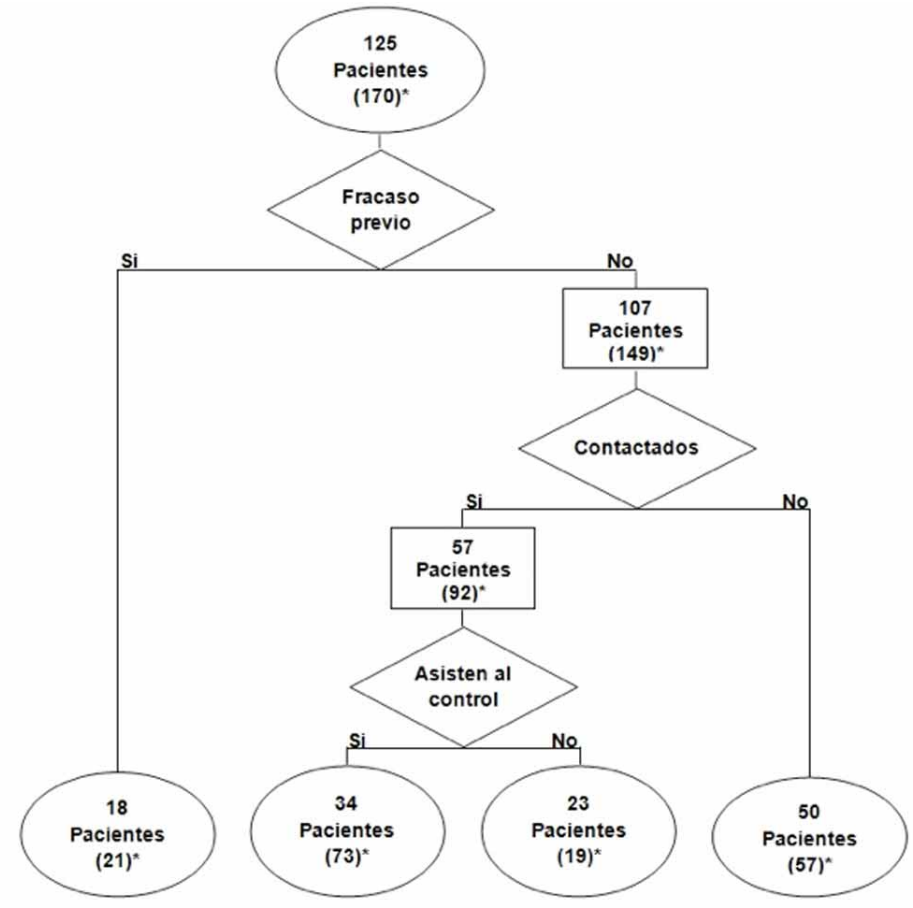

Fig. 1. Diagrama de flujo de pacientes que cumplieron con el criterio de selección.

${ }^{*}$ Cantidad tratamientos de pulpotomías realizados en esos pacientes.

mujeres. Los tratamientos en el arco maxilar corresponden a un $48,8 \%(83 / 170)$ y un $51,2 \%(87 / 170)$ para la mandibular; los dientes más tratados fueron los molares 64 y 74 con un $14,7 \%$ cada uno (Tabla I).

Al analizar las curvas de supervivencia en meses, mediante la prueba Mantel-Cox logrank, no se encontraron diferencias significativas respecto a ninguna de las variables dependientes $(p>0,05)$. Según sexo, la mediana de supervivencia fue mayor en hombres que en mujeres, alcanzando 32 y 30 meses respectivamente. Según el arco dentario, los tratamientos realizados en el maxilar tienen una mediana de supervivencia mayor a la mandibular alcanzando valores de 40 y 30

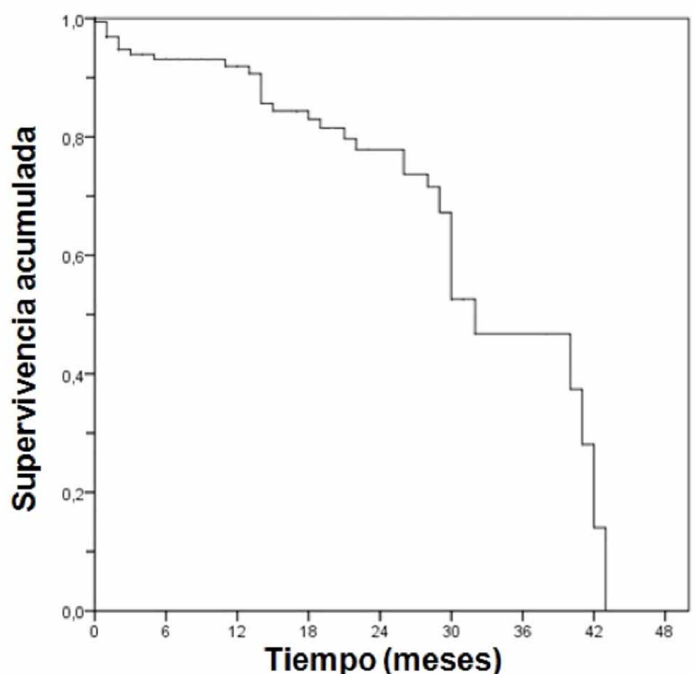

Fig. 2. Función de supervivencia en meses de los pacientes tratados en la Clínica Odontológica UACh en el periodo 2008-20011.

meses respectivamente. En cuanto a los intervalos de edad de los pacientes evaluados, la mediana de supervivencia en meses, el mayor tiempo se encuentra entre los 8-9 años (41 meses) y el menor a los 4-5 años (30 meses) (Tabla II). El método de Kaplan-Meier arrojó una mediana de supervivencia para el total de pulpotomías de 32 meses (Fig. 2).

Tabla II. Mediana de supervivencia según intervalo de edad de los pacientes atendidos en Clínica Odontológica UACh, 2008-2011.

\begin{tabular}{lc}
\hline Edad & $\begin{array}{c}\text { Mediana de } \\
\text { supervivencia }\end{array}$ \\
\hline $\mathbf{4}-\mathbf{5}$ & 30 \\
$\mathbf{6}-\mathbf{7}$ & $\mathrm{N} / \mathrm{D}^{*}$ \\
$\mathbf{8}-\mathbf{9}$ & 41 \\
$\mathbf{1 0}$ & 32 \\
\hline Global & 32 \\
\hline
\end{tabular}

${ }^{*}$ Este dato no se pudo estimar debido a que los dientes restantes todavía están presentes al momento de concluir el estudio.
Tabla I. Relación entre sexo, edad y arco dentario según el número de tratamientos realizados en Clínica Odontológica UACh, 2008-2011.

\begin{tabular}{lccccc}
\hline \multirow{2}{*}{ Edad } & \multicolumn{2}{c}{ Hombres } & \multicolumn{2}{c}{ Mujeres } & \multirow{2}{*}{ Total } \\
\cline { 2 - 5 } & Maxilar & Mandibular & Maxilar & Mandibular & \\
\hline $\mathbf{4 - 5}$ & 9 & 12 & 7 & 15 & 43 \\
$\mathbf{6 - 7}$ & 7 & 17 & 14 & 10 & 48 \\
$\mathbf{8} \mathbf{- 9}$ & 20 & 20 & 20 & 8 & 68 \\
$\mathbf{1 0}$ & 4 & 4 & 2 & 1 & 11 \\
\hline Total & 40 & 53 & 43 & 34 & 170 \\
\hline
\end{tabular}




\section{DISCUSIÓN}

Este estudio fue diseñado para analizar la supervivencia de pulpotomías en molares temporales vitales realizadas por estudiantes de pregrado en la clínica Odontológica de la UACh, y su relación con las variables: sexo, edad y arco en el que se encuentra el diente. No obstante, la vasta diferencia de habilidades que puede existir entre los alumnos podría influir en el tiempo de supervivencia, lo cual se compensaría, en cierta forma, por la supervisión de los tratamientos por parte de docentes especializados y basados en las guías de tratamientos y la evidencia disponible publicadas hasta el momento. La revisión de fichas clínicas fue otra limitante, ya que producto de problemas de legibilidad y omisiones, no pudimos analizar la supervivencia en cuanto a tipo de aislamiento y tipo de restauración definitiva.

Además un $72,8 \%$ no asistió al control del estudio, de esta manera se obtuvo el tiempo de supervivencia basado en el último control registrado en la ficha clínica, lo que pudo haber afectado el tiempo de supervivencia.

El lector debe considerar que el evaluar el éxito de la pulpotomía mediante parámetros clínicos, tal cual hace Hill, basándose en los criterios clínicos de Dean et al. (2002), podría sobreestimar en cierta medida el tiempo de supervivencia, al no considerar criterios radiográficos de éxito.

Al momento de la recolección de datos, se observó una asimetría en la distribución de pacientes que asistieron a controles $(27,2 \%)$, los cuales aportaron con un $42,9 \%$ de los casos, comparado con aquellos que no asistieron a control $(72,8 \%)$, aportando con un $57,1 \%$ de los casos.

Al analizar la variable sexo, no existieron diferencias estadísticamente significativas en cuanto a las curvas de supervivencia, tal cual afirma Kurji et al. (2011) y Guelmann et al. (2002).

En cuanto al arco dentario, tampoco existe diferencia estadísticamente significativa al igual que lo reportado por Guelmann et al. (2002), Kurji et al. sin embargo, reporta que la supervivencia de los dientes mandibulares es menor. Los autores creen que la diferencia de 10 meses encontrada en cuanto a las medianas de supervivencia según arco es significativa clínicamente, lo que pudiese indicar que los dientes mandibulares tienen peor pronóstico.

La edad, tal como dice Hill, es un factor influyente en la sobrevida, evidentemente por la exfoliación de los dientes temporales; Guelmann et al. (2002) establece 2 rangos de edad, menores de 6 años y mayores de 6 años, encontrando en estos últimos menos posibilidades de éxito, Kurji el al. en cambio, reporta que la edad no es un factor influyente. En el presente estudio no se encontraron diferencias estadísticamente significativas al analizar las curvas de supervivencia según edad, pero los autores creen que existe diferencia clínicamente significativa en cuanto a las medianas de supervivencia, encontrando el valor mayor en el intervalo 8-9 años, probablemente porque a los 10 años el tiempo de supervivencia se ve afectado por la cercanía al tiempo de exfoliación tal como infiere Hill; posiblemente en el intervalo de menor edad (4-5 años) influye la dificultad que existe en el manejo clínico del operador (Fuks, 2000; Casas et al., 2003; Farooq et al., 2000) y en el control de higiene que es más difícil a edades tempranas (Unkel et al., 1995).

Analizando la curva general, se observa un buen pronóstico a los 2 años (aproximadamente un $80 \%$ de posibilidades de que el diente permanezca en boca); antes de cumplido el mes existe un $7,6 \%$ de posibilidades de que el tratamiento fracase, lo que los autores atribuyen principalmente a un diagnóstico errado (Vij et al., 2004; Guelmann et al., 2005; Holan et al., 2005; Coll \& Sadrian, 1996). Después de los 32 meses el pronóstico disminuye categóricamente, existiendo menos de un $50 \%$ de posibilidades de éxito. La mayor pendiente en la curva se observa desde el mes 40 , cuando en sólo 3 meses la probabilidad de éxito disminuye de 47 a $0 \%$ por lo que los autores creen que al momento de realizar el tratamiento no debe esperarse una supervivencia mayor a 40 meses.

Hill obtuvo una mediana de supervivencia similar a la del presente estudio, analizando la supervivencia en dientes vitales y no vitales, demostrando que las pulpotomías en dientes no vitales tienen una supervivencia significativamente menor en comparación con los vitales (20,4 contra un 32,7 meses respectivamente), lo que demuestra que es fundamental el acertado diagnóstico pulpar. Según el protocolo clínico de la Universidad Austral de Chile, las pulpotomías se realizan en dientes vitales asintomáticos o con sintomatología reversible tal cual lo señala la guía de la American Academy of Pediatric Dentristy (Coll, 2008; Fuks, 2008). 
Según la revisión de Fuks (2008), que evalúa el éxito de la pulpotomía con distintas técnicas, se observan porcentajes de éxito clínico con rangos para todos los medicamentos de conservación pulpar, exceptuando el hidróxido de calcio, de 90 a $100 \%$ a los 12 meses, de 88 a $100 \%$ a los los 24 meses y a los 36 meses de 97 a 100\%. Sin embargo, se debe considerar que aquellos estudios evalúan el éxito de dichas terapias en un tiempo limitado con criterios clínicos y radiográficos estrictos, como lo es un estudio clínico aleatorizado con factores controlados en condiciones ideales, a diferencia del análisis de supervivencia que estima el tiempo en boca de un diente temporal tratado con pulpotomía en condiciones reales, con criterios de éxito aplicados normalmente en clínica. Tal como afirma Guelmann et al. (2005), normalmente las pulpotomías no son realizadas en condiciones ideales (urgencia, falta de tiempo, paciente no cooperador, limitantes económicas), bajo estas condiciones la tasa de éxito puede bajar significativamente a $53 \%$ en 3 meses. No obstante, la gran baja de tasa de éxito después de un año se atribuye a la microfiltración del material de restauración temporal. Por lo tanto, el éxito de la terapia radica en la capacidad de prevenir microfiltraciones del material restaurador.

A diferencia de Hill los autores consideraron que la exfoliación natural es un éxito clínico, ya que si el diente no hubiese exfoliado, el tratamiento seguiría cumpliendo su objetivo, por lo que se consideró como dato censurado. Además, Hill hizo controles regulares cada un año; nuestros controles en cambio, son irregulares, teniendo una mediana de seguimiento de 9 meses con un rango entre 0 a 43 meses.
Nadin et al. (2003), considera como resultado aceptable de la terapia pulpar, la permanencia asintomática del diente tratado, más de 12 meses; por consiguiente, el pronóstico del diente con pulpotomía es bueno, $y$ al igual que lo que dice la evidencia disponible (Hill) existe un $50 \%$ de posibilidades de que el tratamiento dure al menos 32 meses, cumpliendo así con el objetivo de mantener el diente en boca, lo que sustenta su uso como tratamiento cuyo fin es prevenir problemas mayores que requieran terapias más complejas, más costosas y con problemas adicionales que afecten el desarrollo normal del paciente pediátrico.

Estos resultados deben tomarse con precaución considerando que se trata de un estudio de tipo cohorte retrospectiva, más aún tomando en cuenta sus limitaciones. Sin embargo posee gran validez interna por ser de tipo censal, recomendándose su replicación en otros centros de atención odontológica para generalizar los resultados.

Se sugiere que en el futuro los ensayos clínicos aleatorizados no debiesen enfocarse en la comparación de materiales de medicación, un tema ampliamente estudiado, sino más bien en la identificación de los factores influyentes en el éxito de la pulpotomía.

\section{AGRADECIMIENTOS}

Esta investigación está basada en una tesis realizada como requerimiento para la obtención del grado académico de licenciado en Odontología de la Universidad Austral de Chile, Julio, 2012.

SALINAS, C.; GUIDOTTI, L.; RODRÍGUEZ, C. \& SOTOMAYOR, C. Survival of deciduous molar teeth with pulpotomy treatment. A Kaplan-Meier analysis. Int. J. Odontostomat., 7(3):441-446, 2013.

ABSTRACT: The aim of this study was to estimate the survival of deciduous molars, treated with pulpotomy at the Dental Clinic of the Universidad Austral de Chile, and its relation with age, sex and dental arch. The design was a retrospective cohort study. A census was made in which all patients of 4 to 10 years old treated at the Dental Clinic were included. Clinical records of patients treated between March 2008 until June 2011 were reviewed. In this study 125 patients with a total of 170 pulpotomies were included. A clinical exam was provided for those patients in which the pulpotomy had not failed. The patients who did not participate in this exam were considered censored, and the survival time was set from the date that the pulpotomy was performed to the last examination recorded in the clinical file. The analysis and tabulation was carried out the statistic program IBM SPSS Statistics 19. The median survival time was obtained through Kaplan Meier method. To determine the difference between age intervals, sex and dental arch the Mantel-Cox logrank method was used ( $p<0.05$ ). The median survival time is 32 months. There was no statistical significant difference between the age intervals, sex and dental arch. Pulpotomy prognosis is good since it establishes a $50 \%$ probability of the molar remaining in the mouth at least 32 months, thereby fulfilling the aim of avoiding premature loss of the molar and related consequences.

KEY WORDS: pulpotomy, deciduous teeth, survival analysis. 


\section{REFERENCIAS BIBLIOGRÁFICAS}

Alaçam, A.; Odabas, M. E.; Tüzüner, T.; Sillelioglu, H. \& Baygin, O. Clinical and radiographic outcomes of calcium hydroxide and formocresol pulpotomies performed by dental students. Oral Surg. Oral Med. Oral Pathol. Oral Radiol. Endod., 108(5):e127-33, 2009.

Casas, M. J.; Layug, M. A.; Kenny, D. J.; Johnston, D. H. \& Judd, P. L. Two-year Outcomes of Primary Molar Ferric Sulfate Pulpotomy and Root Canal Therapy. Pediatr. Dent., 25(2):97-102, 2003.

Coll, J. A. \& Sadrian, R. Predicting pulpectomy success and its relationship to exfoliation and succedaneous dentition. Pediatr. Dent., 18(1):57-63, 1996.

Coll, J. A. Indirect pulp capping and primary teeth: is the primary tooth pulpotomy out of date? Pediatr. Dent., 30(3):230-6, 2008.

Cordeiro, M. M. \& Rocha, M. J. The effects of periradicular inflamation and infection on a primary tooth and permanent successor. J. Clin. Pediatr. Dent., 29(3):193200, 2005

Dean, J. A.; Mack, R. B.; Fulkerson, B. T. \& Sanders B.J. Comparison of electrosurgical and formocresol pulpotomy procedures in children. Int. J. Paediatr. Dent., 12(3):177-82, 2002.

Farooq, N. S.; Coll, J. A.; Kuwabara, A. \& Shelton, P. Success rates of formocresol pulpotomy and indirect pulp therapy in the treatment of deep dentinal caries in primary teeth. Pediatr. Dent., 22(4):278-86, 2000.

Fuks, A. B. Pulp Therapy for the Primary and Young Permanent Dentitions. Dent. Clin. North Am., 44(3):57196, vii, 2000.

Fuks, A. B. Vital pulp therapy with new materials for primary teeth: new directions and Treatment perspectives. Pediatr. Dent., 30(3):211-9, 2008.

Guelmann, M.; Fair, J.; Turner, C. \& Courts, F. J. The success of emergency pulpotomies in primary molars. Pediatr. Dent., 24(3):217-20, 2002.

Guelmann, M.; Fair, J. \& Bimstein, E. Permanent Versus Temporary Restorations after Emergency Pulpotomies in Primary Molars. Pediatr. Dent., 27(6):478-81, 2005.

Hill, M. W. The survival of vital and non-vital deciduous molar teeth following pulpotomy. Aust. Dent. J., 52(3):1816, 2007.

Holan, G.; Eidelman, E. \& Fuks, A. B. Long-term evaluation of pulpotomy in primary molars using mineral trioxide aggregate or formocresol. Pediatr. Dent., 27(2):129-36, 2005.

Huth, K. C.; Paschos, E.; Hajek-Al-Khatar, N.; Hollweck, R.; Crispin, A.; Hickel, R. \& Folwaczny, M. Effectiveness of 4 pulpotomy techniques--randomized controlled trial. J. Dent. Res., 84(12):1144-8, 2005.

Koshy, S. \& Love, R.M. Endodontic treatment in the primary dentition. Aust. Endod. J., 30(2):59-68, 2004.

Kurji, Z. A.; Sigal, M. J.; Andrews, P. \& Titley, K. A retrospective study of a modified 1-minute formocresol pulpotomy technique part 1: clinical and radiographic findings. Pediatr. Dent., 33(2):131-8, 2011.

Nadin, G.; Goel, B. R.; Yeung, C. A. \& Glenny, A. M. Pulp treatment for extensive decay in primary teeth. Cochrane Database Syst. Rev., (1):CD003220, 2003.

Unkel, J. H.; Fenton, S. J.; Hobbs, G. Jr. \& Frere, C. L. Toothbrushing ability is related to age in children. ASDC J. Dent. Child., 62(5):346-8, 1995.

Vij, R.; Coll, J. A.; Shelton, P. \& Farooq, N. S. Caries control and other variables associated with success of primarymolar vital pulp therapy. Pediatr. Dent., 26(3):21420, 2004.

Von Elm, E.; Altman, D. G.; Egger, M.; Pocock, S. J.; Gøtzsche, P. C.; Vandenbroucke, J. P. \& STROBE Initiative. The Strengthening the Reporting of Observational Studies in Epidemiology (STROBE) statement: guidelines for reporting observational studies. Ann. Intern. Med., 147(8):573-7, 2007.

Waterhouse, P. J.; Nunn, J. H.; Whitworth, J. M. \& Soames, J. V. Primary molar pulp therapy--histological evaluation of failure. Int. J. Paediatr. Dent., 10(4):313-21, 2000.

Dirección para Correspondencia:

Dra. Carolina Rodríguez Salinas

Docente Adjunto Clínica del Niño

Facultad de Medicina, Escuela de Odontología

Universidad Austral de Chile, Valdivia

CHILE

Email: carodsa@gmail.com

Recibido : 10-07-2012

Aceptado: 09-10-2013 